\title{
ON THE EXISTENCE OF SLICES FOR TRANSFORMATION GROUPS
}

\author{
HSIN $\mathrm{CHU}^{1}$
}

1. Introduction. One of the most interesting results of transformation groups is the existence of slices. If the group is compact and Lie and the space is completely regular, Mostow proved [5], as a generalization of works of Gleason, Koszul, Montgomery, Yang and many others, that there is a $G_{x}$-slice through every $x$ in the space where $G_{x}$ is the isotropy subgroup at $x$. If the group is not compact but Lie and if the space is a Cartan space, then Palais proves in [7] the same result. As we know, if the space is Cartan then each orbit of the space is closed and each isotopy subgroup is compact. From the point of view of ordinary differential equations, Dugundji and Antosiewicz study (see [2]) parallelizable flow where the space is metric and the group is the real line. The parallelizable flow is essentially a trivial bundle and a special Cartan space. Consequently, it has a $G_{x}$-slice for every $x$. If the space is not Cartan, the situation is very different and it need not have $G_{x}$-slices (see [7]). In this note we show that some of these spaces do have slices of certain type for each point.

Let $(X, T, \Pi)$ be a transformation group where $T$ is a noncompact Lie group. Let $S$ be a closed, normal, syndetic subgroup of $T$. If $X$ is locally compact, normal, Hausdorff and $(X, S, \Pi)$ is locally weakly almost periodic, then through each $x \in X$, we show that there is an $H_{x}$-slice, where $H_{x}$ is a closed subgroup of $G$, and contains $G_{x}$. In general, the sets $H_{x}$ and $H_{x}$-slice can be chosen as proper subsets of $T$ and $X$ respectively if $\mathrm{Cl}(x S) \neq \mathrm{Cl}(x T)$. We also show that if $T$ is any topological group and $X$ is compact, Hausdorff, minimal but not minimal under a closed normal, syndetic subgroup $S$ of $T$ then there is a proper closed subgroup $H$ of $T$ such that for each $x \in X$ there is a proper closed subset $B_{x}$ of $X$ such that $B_{x}$ is a global $H$-slice. In fact, $H=\{h \mid x h \in \mathrm{Cl}(x S)\}$ and $B_{x}=\mathrm{Cl}(x H)$ for $x \in X$.

2. Lemmas. We may find the following definitions in [3].

Definition. Let $(X, T, \Pi)$ be a transformation group. Let $x \in X$. The group $T$ is called locally weakly almost periodic at $x$ if $U$ is a neighborhood of $x$, there exist a neighborhood $V$ of $x$ and a compact

Received by the editors February 7, 1966.

1 This work was partially supported by contract NAS 8-1646 with the George C. Marshall Space Flight Center, NASA, and Grant GP-6167 with the National Science Foundation. 
subset $K$ of $T$ such that $y \in V$ and $t \in T$ imply $y t K \cap U \neq \varnothing$. The group $T$ is called locally weakly almost periodic on $X$ if $T$ is locally weakly almost periodic at every $x \in X$. The space $X$ is called minimal under $T$ if $\mathrm{Cl}(x T)=X$ for every $x \in X$.

Lemma 1. Let $(X, T, \Pi)$ be a transformation group, where $X$ is a. locally compact, Hausdorff space and $T$ is locally weakly almost periodic. Then the class of all orbit-closures under $T$ is a star-open and star-closed partition and each orbit-closure is compact.

Proof. By a star-open (star-closed) partition, we mean it is a partition of the space $X$ and for every open set (closed set) $V$ of $X$, the set $V^{*}=\{\mathrm{Cl}(x T) \mid x \in V\}$ is open (closed) (see [3]). This lemma is known. For example, it is the direct consequence of Remark 2.30, Theorem 4.15 and Theorem 4.16 in [3].

Lemma 2. Let $(X, T, \Pi)$ be a transformation group, where $X$ is a compact, Hausdorf, minimal set. Let $S$ be a closed syndetic normal subgroup of $T$ such that $X$ is not minimal under $S$. Then the class of all orbit-closures under $S$ is a star-open and star-closed partition and each orbit-closure is compact.

Proof. It is a consequence of Lemma 1 and the fact that in this case $S$ is locally weakly almost periodic on $X$ (see Theorem 4.19 in [3]). By a syndetic subgroup, we mean $S \cdot K=T$ for some compact set $K$ in $T$.

Let $(X, T, \Pi)$ be a locally weakly almost periodic transformation group, where $X$ is a locally compact and Hausdorff space and $T$ is a Lie group. Let $S$ be a closed, normal, syndetic subgroup of $T$. Let $R$ be the relation on $X$ defined by the orbit-closures of $S$, namely $x R y$ if and only if $x \in \mathrm{Cl}(y S)$. We know that $x \in \mathrm{Cl}(y S)$ iff $y \in \mathrm{Cl}(x S)$ (see [3]); it is clear that $R$ is an equivalence relation.

Lemma 3. If $S$ is locally weakly almost periodic on $X$, then $R$ is an open and closed relation and the quotient space $X^{*}=X / R$ is Hausdorff and locally compact. If $X$ is normal then $X^{*}$ is normal.

Proof. Let $P: X \rightarrow X^{*}$ be the natural projection. Then by Lemma $1, P$ is open and closed. We shall show that $X^{*}$ is Hausdorff. Let $x^{*}=P(\mathrm{Cl}(x S))$ and $y^{*}=P(\mathrm{Cl}(y S))$ be two distinct points in $X^{*}$. Then, by Lemma $1, \mathrm{Cl}(x S)$ and $\mathrm{Cl}(y S)$ are compact and $\mathrm{Cl}(x S) \cap \mathrm{Cl}(y S)=\varnothing$. Since $X$ is Hausdorff and $\mathrm{Cl}(x S)$ and $\mathrm{Cl}(y S)$ are compact, there exist two open sets $U$ and $V$ such that $\mathrm{Cl}(x S) \subset U$ and $\mathrm{Cl}(y S) \subset V$ with $U \cap V=\varnothing$. Consequently, $\bar{U} \cap \mathrm{Cl}(y S)=\varnothing$. Let $U^{\prime}=\{\mathrm{Cl}(z S) \mid z \in U\}$ and $(\bar{U})^{\prime}=\{\mathrm{Cl}(z S) \mid z \in \bar{U}\}$. It is easy to see that 
$(\bar{U})^{\prime} \cap \mathrm{Cl}(y S)=\varnothing$. Then $P\left(U^{\prime}\right)=U^{*}$ is open in $X^{*}$ and $P\left((\bar{U})^{\prime}\right)$ $=(\bar{U})^{*}$ is closed in $X^{*}$ with $x^{*} \in U^{*}$ and $y^{*} \in \bar{U}^{*}$. Let $W^{*}=X^{*}$ $-(\bar{U})^{*}$. Then $W^{*}$ is open in $X^{*}, y^{*} \in W^{*}$ and $W^{*} \cap U^{*}=\varnothing$. Since $P$ is open and continuous, $X^{*}$ is also locally compact if $X$ is locally compact. Similarly, we can show $X^{*}$ is normal if $X$ is normal. The Lemma is proved.

Let $T^{*}=T / S$ be the quotient group of $T$. It is again a Lie group. Denote $q: T \rightarrow T^{*}$ to be the natural projection. Define $\Pi^{*}: X^{*} \times T^{*}$ $\rightarrow X^{*}$ by $\Pi^{*}\left(x^{*}, t^{*}\right)=(x t)^{*}$.

LEMмa 4. The following diagram:

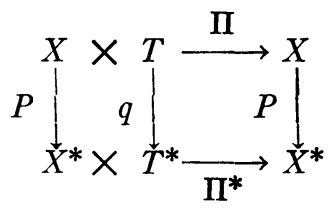

is commutative and the triple $\left(X^{*}, T^{*}, \Pi^{*}\right)$ is a transformation group induced by $(X, T, \Pi)$.

Proof. To show the diagram is commutative it is enough to show that for each $x \in X$ and $t \in T, P(\mathrm{Cl}(x S) S t)=(x t)^{*}$. Since $\mathrm{Cl}(x S) S t$ $=\mathrm{Cl}(x S) t=\mathrm{Cl}((x t) S)$ we have $P(\mathrm{Cl}((x t) S))=(x t)^{*}$. That the mapping $\Pi^{*}$ is continuous is clear from the fact that both $p$ and $q$ are open and continuous. To show that $\left(X^{*}, T^{*}, \Pi^{*}\right)$ is a transformation group is a straightforward computation by using the facts: $p$ and $q$ are open and continuous, $S$ is a closed normal subgroup and the above diagram is commutative.

3. Main results. We may find the following definition from [6]:

Definition. Let $H$ be a closed subgroup of $T$. A subset $B$ of $X$ is called an $H$-kernel if

(1) $B$ is closed in $B T$,

(2) $B H=B$,

(3) for each $t \in T-H, B t \cap B=\varnothing$.

An $H$-kernel $B$ in $X$ is called an $H$-slice in $X$ if $B T$ is open. If $B T=X$, we call $B$ a global $H$-slice for $X$. If $x \in X$ then by a slice at $x$ we mean a $G_{x}$-slice in $X$.

THEOREM 1. Let $(X, T, \Pi)$ be a locally weakly almost periodic transformation group, where $X$ is a locally compact, normal, Hausdorff space and $T$ is a noncompact Lie group. Let $S$ be a proper, closed, normal, syndetic subgroup of $T$. If $X$ is not a minimal set under $S$, then for each $x \in X$, there is a closed subgroup $H_{x}$ such that $\left(S \cup G_{x}\right) \subset H_{x}$ and a closed 
subset $B_{x}$ such that $B_{x}$ is an $H_{x}$-slice in $X$. In general, if $\mathrm{Cl}(x S) \neq \mathrm{Cl}(x T)$ for $x \in X$, the sets $H_{x}$ and $B_{x}$ can both be chosen as proper subsets of $T$ and $X$ respectively.

Proof. Let $\left(X^{*}, T^{*}, \Pi^{*}\right)$ be the induced transformation group of $(X, T, \Pi)$ as in Lemma 4 . Then $X^{*}$ is again locally compact, normal, Hausdorff and $T^{*}$ is a compact Lie group. Let $x \in X$ and $p(x)=x^{*}$. Since $X^{*}$ is complete regular, by a known result as we quoted in the

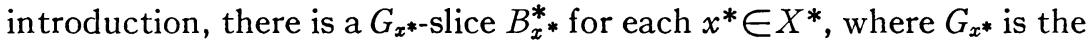
isotropy subgroup of $T^{*}$ of $x^{*}$. Let $p^{-1}\left(x^{*}\right)=\mathrm{Cl}(x S)$ for the given $x \in X$. Let $q^{-1}\left(G_{x^{*}}\right)=H_{x}$. Then $H_{x}$ is a closed subgroup of $T$ which contains $G_{x}$ as well as $S$. Let $p^{-1}\left(B_{x^{*}}^{*}\right)=B$. Let $p^{-1}\left(B_{x}^{*} T^{*}\right)=M$. It is clear that $B_{x}$ is invariant under $S$ and $M=B_{x} T$. The openness of $B_{x}^{*} T^{*}$ in $X^{*}$ implies the openness of $M$ in $X$. We know $B_{x^{*}}^{*}$ is closed in $B_{x *}^{*} T^{*}$, it follows that $B_{x}$ is closed in $B_{x} T$. From $B_{x^{*}}^{*} G_{x^{*}}=B_{x^{*}}^{*}$ we have $B_{x} H_{x}=B_{x}$. It remains to show that for each $t \in T-H_{x}, B_{x} t \cap B_{x}=\varnothing$. Let $q(t)=t$, then $q^{-1}(t)=t S$. It is clear that $t^{*} \in T^{*}-G_{x^{*}}$. Consequently, $B_{x}^{*} t^{*} \cap B_{x^{*}}^{*}=\varnothing$. It implies that $B_{x} t S \cap B_{x}=\varnothing$. From $B_{x} t S$ $=B_{x} S t=B_{x} t$, we have $B_{x} t \cap B_{x}=\varnothing$. This proves that there is an $H_{x^{-}}$ slice for every $x \in X$.

If $\mathrm{Cl}(x S) \neq \mathrm{Cl}(x T)$, then $G_{x^{*}} \neq T^{*}$ and, from the properties of a slice, $B^{*} \neq X$. Consequently, $p^{-1}\left(B^{*}\right)=B$ and $q^{-1}\left(G_{x^{*}}\right)=H_{x}$ are proper subsets of $X$ and $T$ respectively.

Theorem 2. Let $(X, T, \Pi)$ be a minimal but not minimal under a closed, normal syndetic subgroup $S$ of $T$, where $X$ is compact Hausdorff and $T$ is any topological group. Then there is a proper closed subgroup $H$ such that for each $x \in X$ there exists a proper closed subset $B_{x}$ with $B_{x}$ as a global $H$-slice in $X$ and $\left(S \cup G_{x}\right) \subset H$. In fact, $H=\{h \mid x h \in \mathrm{Cl}(s S)\}$ and $B_{x}=\mathrm{Cl}(x H)$ for every $x \in X$.

Proof. It is known, in this case, that the class of all orbit-closures under $S$ is a decomposition of $X$ (e.g. see [3, p. 33]). In particular, $\mathrm{Cl}(x S)$ is a minimal set under $S$ and $\mathrm{Cl}(x S) \neq X$ for every $x \in X$. Let $B_{x}=\mathrm{Cl}(x S)$ and $H=\{t \mid x t \in \mathrm{Cl}(x S), t \in T\}$. Since $S$ is normal and $\mathrm{Cl}(x S)$ is minimal under $S$ it is not hard to see that $H$ is a proper, closed subgroup of $T$. We shall see that $\mathrm{Cl}(x S)$ is a global $H$-slice at $x$ in $X$. We know that (1) $\mathrm{Cl}(x S) T=\mathrm{Cl}(x T)=X$ and $\mathrm{Cl}(x S)$ is, of course, closed in $X$; (2) for each $h \in H$, we have $x h \in \mathrm{Cl}(x S)$, it implies $\mathrm{Cl}(x S) h=\mathrm{Cl}(x h S)$. Since $\mathrm{Cl}(x S)$ is a minimal set under $S$, we have $\mathrm{Cl}(x h S)=\mathrm{Cl}(x S)$ or $\mathrm{Cl}(x S) H=\mathrm{Cl}(x S)$; (3) from the definition of $H$ we have for each $t \in T-H, \mathrm{Cl}(x S) t \cap \mathrm{Cl}(x S)=\varnothing$. Suppose not. There are $y$ and $z$ in $\mathrm{Cl}(x S)$ such that $y t=z$ or $\mathrm{Cl}(y t S)=\mathrm{Cl}(z S)$. This implies 
$\mathrm{Cl}(y S) t=\mathrm{Cl}(z S)$. Since $\mathrm{Cl}(x S)=\mathrm{Cl}(y S)=\mathrm{Cl}(z S)$ we have $\mathrm{Cl}(x S) t$ $=\mathrm{Cl}(x S)$ or $x t \in \mathrm{Cl}(x S)$ and $t \in H$. A contradiction! We already show that $\mathrm{Cl}(x S) t=X$. Consequently, $\mathrm{Cl}(x S)$ is a global $H$-slice at $x$.

For each $g \in G_{x}$, we have $x g=x \in \mathrm{Cl}(x S)$, and it follows that $G_{x} \subset H$. It is also easy to see that $S \subset H$. The theorem is proved.

The most known examples of minimal continuous flows are not totally minimal, including nilflows (see [1]).

\section{REFERENCES}

1. L. Auslander, L. Green and F. Hahn, Flows on homogeneous spaces, Annals of Mathematics Studies, No. 53, Princeton Univ. Press, Princeton, N. J., 1963.

tof 2. J. Dugundji and H. A. Antosiewicz, Parallelizable flows and Lyapunov's second method, Ann. of Math. 73 (1961), 543-555.

3. W. H. Gottschalk and G. A. Hedlund, Topological dynamics, Colloq. Publ., Vol. 36, Amer. Math. Soc., Providence, R. I., 1955.

4. D. Montgomery and C. T. Yang, The existence of a slice, Ann. of Math. 65 (1957), 108-116.

5. G. D. Mostow, Equivariant imbedding in Euclidean space, Ann. of Math. 65 (1957), 432-446.

6. R. S. Palais, The classification of G-spaces, Mem. Amer. Math. Soc. No. 36, (1960), 72 pp.

7. - On the existence of slices for actions of non-compact Lie groups, Ann. of Math. 73 (1961), 295-323.

UNIVERSITY OF MARYLAND 